\title{
Classification des cycles d'une usine marémotrice
}

\section{The classifying of tidal plant cycles}

\author{
PAR M. CASEAU,

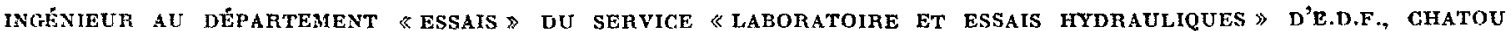

\begin{abstract}
A partir de l'étude des différents types de marche de l'usine, on aborde le problème général qui est l'optimisation de l'exploitation sur une période donnée. On démontre alors qu'il n'y a qu'un nombre fini de choix possibles entre les diverse marches et cela dans des conditions bien déterminées. On peut ainsi dénombrer les systèmes de choix possibles qui donnent chacun naissance da un cycle unique. Ce dénombrement effectué, on classera les cycles par familles en tâchant d'éliminer a priori le plus grand nombre de cycles inintéressants.
\end{abstract}

Starting out from an analysis of the different types of power plant operation, the general problem of optimising the running of the plant over a given period is approached. It is then shown that the number of possible choices between various forms of operation is finite, this under well-determined conditions. The numbers of possible systems of choice each resulting in a unique cycle can thus be determined and the cycles classified according to families, whilst discarding, for the time being, as many of the irrelevant cycles as possible.

PREMIERE PARTIE

\section{LES DIFFÉRENTS TYPES DE FONCTIONNEMENT}

\section{ETUDE GENERALE}

Rappelons d'abord qu'il y a six types de marche possibles, que nous allons classer :

(0) Arrêt : le niveau du bassin reste constant;

(1) Turbine directe - Pompe inverse,

$\left(1^{\prime}\right)=(1)+$ Vannage;

(2) Turbine inverse - Pompe directe,

$\left(2^{\prime}\right)=(2)+$ Vannage;

(3) Arrêt sur la courbe de marée - La puissance est nulle et le niveau du bassin est le même que celui de la mer.

Ces six types de marche correspondent à six types d'équation possibles. Remarquons en effet que la «colline » $\mathbf{N}(q, \mathrm{H})$ se décompose en deux nappes distinctes sans communication possible $\mathrm{N}_{1}(q, \mathrm{H})$ (trajet $(1), q>0$ ) et $\mathrm{N}_{2}(q, \mathrm{H})$ (trajet (2) $q<0)$. Au contraire, il n'y a aucune raison 


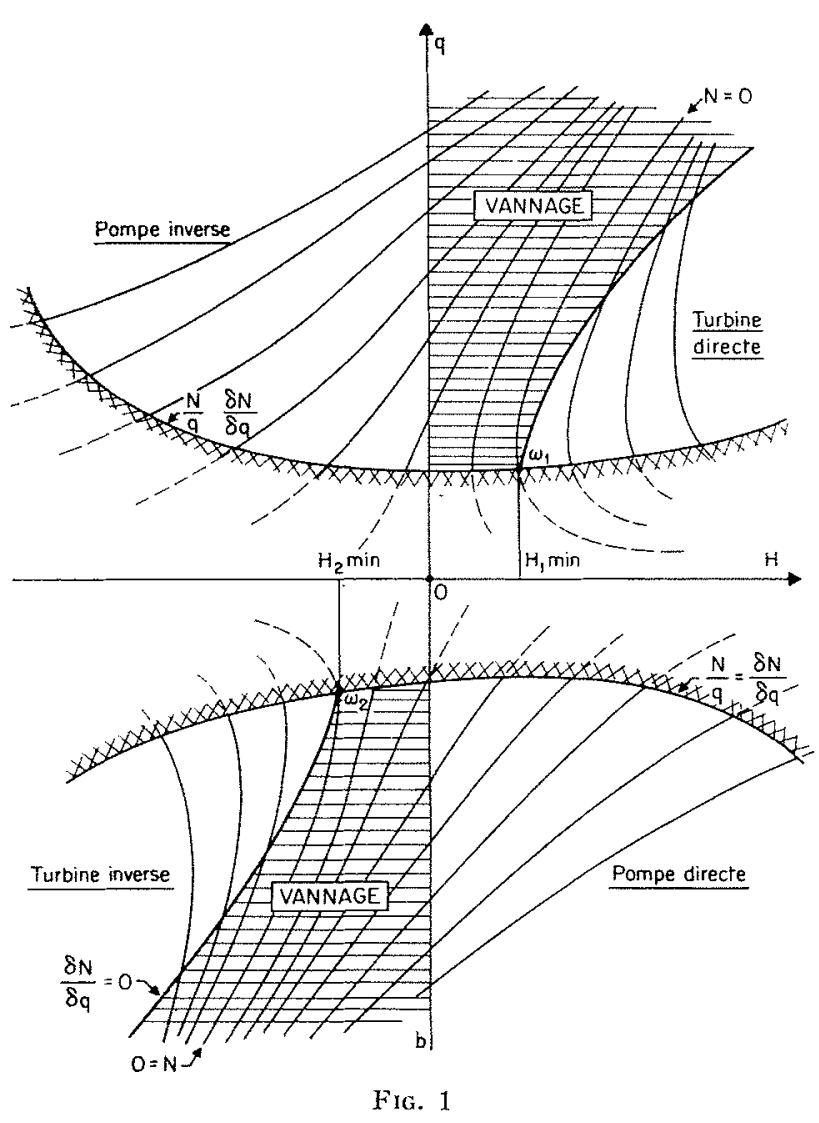

de distinguer entre turbine et pompe, la surface $N_{1}(q, H)$ est régulière sur l'ensemble du domaine des $\mathrm{H}$, positifs ou négatifs.

Classons nos six types de marche avec leurs caractéristiques les plus importantes :

\begin{tabular}{|c|c|c|c|}
\hline$(0)$ & $q=0$ & $q_{V}=0$ & $\mathrm{~N}=0$ \\
\hline$(1)$ & $q>0$ & $q_{V}=0$ & Colline $\mathrm{N}_{1}$ \\
\hline$\left(1^{\prime}\right)$ & $q>0$ & $q_{V}=\mathrm{K} \sqrt{\mathrm{H}}$ & Colline $\mathrm{N}_{1}$ \\
\hline$(2)$ & $q<0$ & $q_{V}=0$ & Colline $\mathrm{N}_{2}$ \\
\hline$\left(2^{\prime}\right)$ & $-q<0$ & $q_{V}=\mathrm{K} \sqrt{\mathrm{H}}$ & Colline $\mathrm{N}_{2}$ \\
\hline$(3)$ & $\mathrm{H}=0$ & $\mathrm{Z}=h(t)$ & $\mathrm{N}=0$ \\
\hline
\end{tabular}

Lorsque l'on a choisi un type de marche, les équations du mouvement sont parfaitement déterminées. Pour (0) et (3), elles sont évidentes et pour $(1),\left(1^{\prime}\right),(2)$ et $\left(2^{\prime}\right)$, ce sont les équations d'Euler [1] exprimant que le trajet considéré est optimal par rapport aux trajets de la même famille.
Nous devons donc étudier le problème le plus général, à savoir : trouver les conditions pour qu'un trajet du type $0,1,1^{\prime} \ldots 3$ soit optimal par rapport aux trajets d'un type quelconque 0 , 1,1 , ... 3. Certains trajets étant, par nature, incompatibles, nous dresserons la table de comparaison :

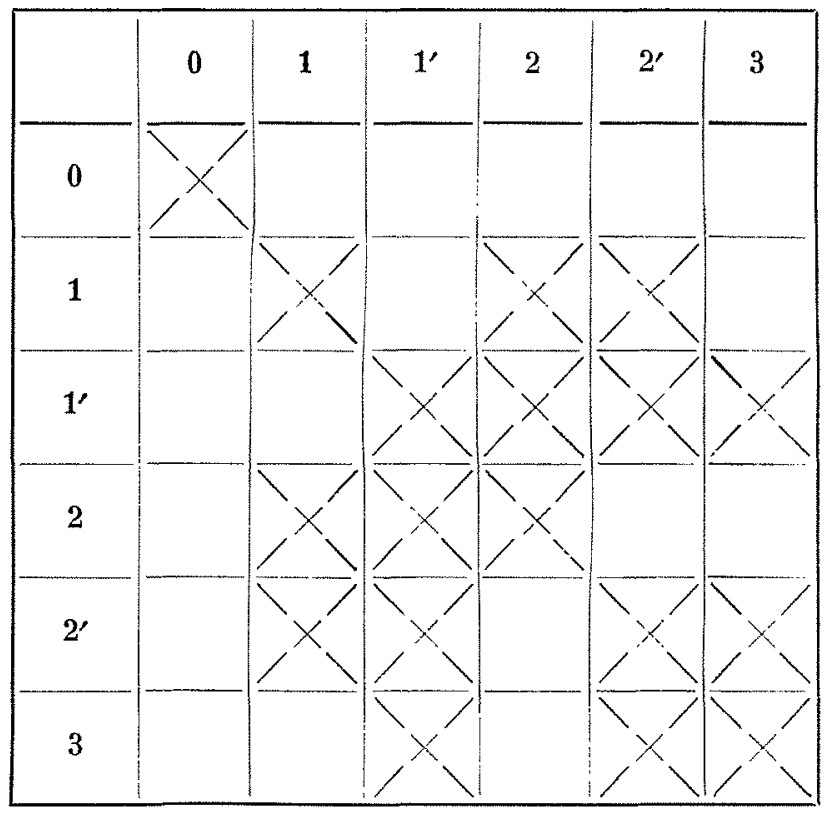

Pour les dix-huit cas restant à étudier, nous allons obtenir des conditions nécessaires et suffisantes, sous forme d'inégalités: elles découpent sur les collines $\mathrm{N}_{1}$ et $\mathrm{N}_{2}$ des zones sur lesquelles le type de trajet est fixé. La plupart d'entre elles ne seront pas nouvelles, et nous passerons dessus assez rapidement, sans donner beaucoup de démonstrations. Ce qui est nouveau, c'est que nous aurons ainsi trouvé les conditions pour qu'un trajet soit optimal par rapport aux trajets d'un type différent.

\section{A - Comparaison $(1) \longleftrightarrow\left(1^{\prime}\right)$ ou $(2) \longleftrightarrow\left(2^{\prime}\right)$.}

On démontre facilement [1] [2] les propositions suivantes :

- Si $\left(\partial N_{1} / \partial q\right)>0$, (1) est optimal par rapport à $\left(1^{\prime}\right)$. Il est interdit de vanner.

-. Si $\left(\partial N_{1} / \partial q\right)<0,\left(1^{\prime}\right)$ est optimal par rapport à (1). Il faut vanner au vannage maximal.

- Lorsqu'une extrémale atteint la frontic̀re $\left(\partial \mathrm{N}_{1} / \partial q\right)=0$, elle la franchit toujours, et on doit changer de type de marche.

Les mêmes propositions sont vraies pour les marches (2) et $\left(2^{\prime}\right)$. 
$B$ - Comparaison (I) ou $\left(1^{\prime}\right) \rightarrow(0)$

et $(2)$ ou $\left(2^{\prime}\right) \rightarrow(0)$.

On démontre de même, les propositions suivantes :

$-\mathrm{Si} N-q(\partial \mathrm{N} / \partial q)=0,(1)$ ou $\left(1^{\prime}\right)$ sont optimaux par rapport à $(0)$;

- Au contraire, les trajets (1) ou (1'), pour lesquels $\mathrm{N}-q(\partial \mathrm{N} / \partial q)<0$, ne sont pas optimaux. On a intérêt à s'arrêter et à passer en $(0)$;

- Lorsqu'une extrémale atteint la frontière $\mathrm{N}-q(\partial \mathrm{N} / \partial \mathrm{q})=\mathbf{0}$, elle la franchit et on doit s'arrêter.

$$
\begin{gathered}
\text { C-Comparaison }(0) \rightarrow(1) \text { ou }\left(1^{\prime}\right) \\
\text { et }(0) \rightarrow(2) \text { ou }\left(2^{\prime}\right) .
\end{gathered}
$$

Nous avons vu que les arrêts ou départs se font sur la courbe $(N / q)=(\partial N / \partial q)$. Sur cette courbe, $(N / q)$ est une fonction de $H$.

Nous admettrons alors (fig, 2) que :

$$
\frac{\mathrm{N}_{1}}{q_{1}} \text { et } \frac{\mathrm{N}_{2}}{q_{2}}
$$

sont des fonctions croissantes.

$$
\frac{\mathrm{N}_{1}}{q_{1}}<\frac{\mathrm{N}_{2}}{q_{2}}
$$

(égalité exclue).

On démontre alors facilement que :

- Un arrêt et un départ se font toujours en sens opposé;

- On a la relation $(\mathrm{N} / q)_{\mathrm{A}}=(\mathrm{N} / q)_{\mathrm{B}}$.

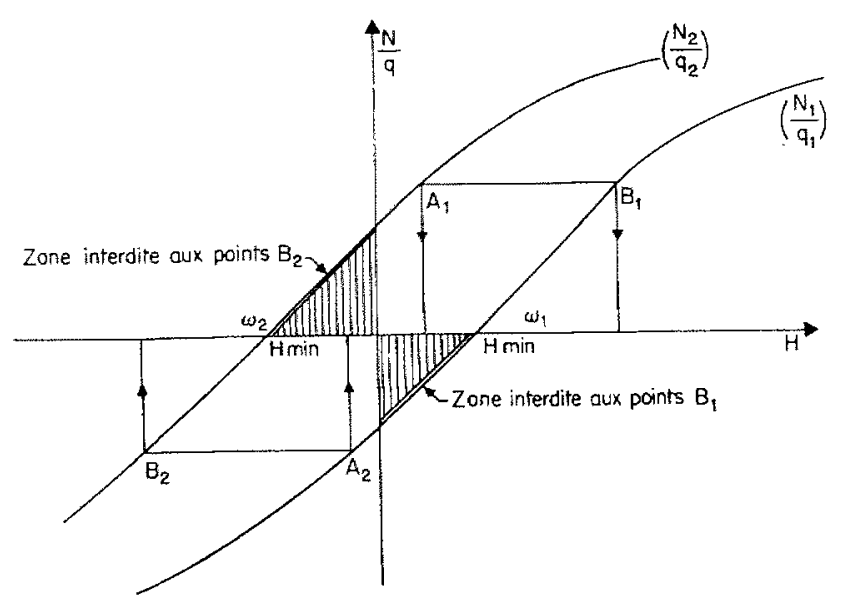

FIG. 2

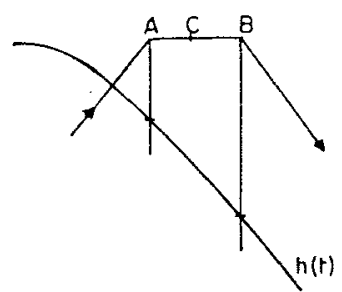

FIG. 3

Soit alors (fig. 3) un segment d'arrêt A correspondant à $\mathrm{N}_{2}, \mathrm{~B}$ correspondant à $\mathrm{N}_{1}, \mathrm{C}$ un point entre $A$ et $B$. On démontre que :

Proposition (1).

On a nécessairement :

$$
\mathrm{H}_{\mathrm{A}} \leqslant \mathrm{H}_{\mathrm{C}} \leqslant \mathrm{H}_{\mathrm{B}} \quad \text { d'où } h_{\mathrm{A}} \geqslant h_{\mathrm{C}} \geqslant h_{\mathrm{B}}
$$

avec, de plus,

$$
\mathrm{H}_{\mathrm{A}}<\mathrm{H}_{\mathrm{B}} \quad \text { et } h_{\mathrm{A}}>h_{\mathrm{B}}
$$

\section{Corollaires :}

Proposition (2).

Sur l'intervalle $\mathrm{AB}, h$ ne peut passer par aucun maximum, il n'y a donc ni crête, ni creux.

Proposition (3).

Soient $(\alpha \beta)$ l'intervalle séparant une crête d'un creux, (AB) un intervalle d'arrêt.

On a nécessairement :

$$
(\mathrm{AB}) \Upsilon(\alpha \beta)=\varnothing
$$

ou bien :

$$
(\mathrm{AB}) \subset(\alpha \beta)
$$

\section{Proposition (4).}

Les pentes vérifient certaines inégalités :

si $(d h / d t)<0$, les départs sont en (1), les arrivées en (2);

si $(d h / d t)>0$, les départs sont en (2), les arrivées en (1).

\section{Proposition (5).}

Sur l'intervalle $(\alpha \beta)$, il ne peut $y$ avoir qu'un seul intervalle $(\mathrm{AB})$.

Démonstration :

Supposons $h$ \. Supposons deux intervalles (AB) $\left(\mathrm{A}^{\prime} \mathrm{B}^{\prime}\right)$ qui, d'après $\left(1^{\prime}\right)$, sont inclus dans 


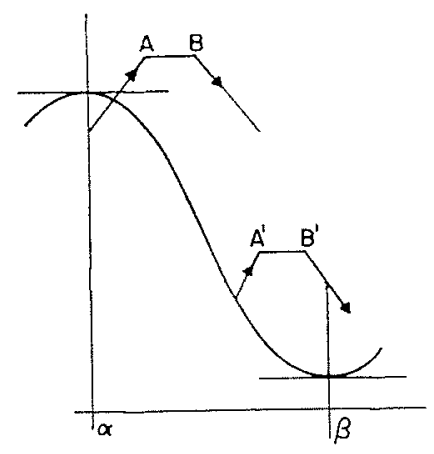

FYG. 4

( $\alpha \beta)$. Les départs sont en (1), les arrivées en (2). On a donc (fig. 4) un départ en $B$ sur (1) et une arrivée en $A^{\prime}$ sur (2), ce qui est impossible.

\section{D - Comparaison $\left(1^{\prime}\right) \rightarrow(0)$ et $\left(2^{\prime}\right) \rightarrow(0)$.}

Les raisonnements précédents et les conditions nécessaires restent inchangés. Donc on a toujours :

$$
\left(\frac{\mathrm{N}}{q}\right)_{\mathrm{A}}=\left(\frac{\mathrm{N}}{q}\right)_{\mathrm{B}}
$$

On voit donc sur la figure 2 qu'il faut vanner si $(\mathrm{N} / q)<0$ et $\mathrm{H}$ positif. Ceci se produit uniquement lorsque (fig. 5) :

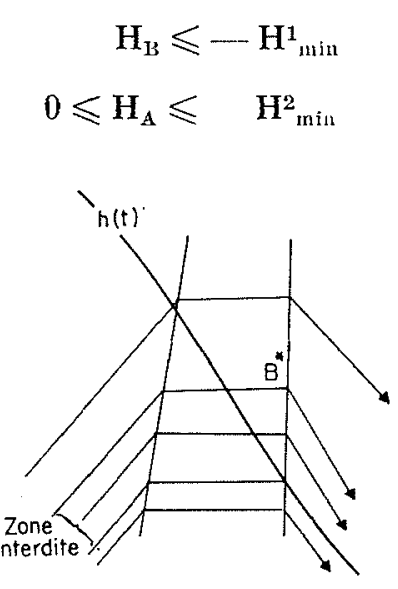

Fig. 5
Le segment $\mathrm{AB}$ coupe alors la courbe $\mathrm{H}=0$. Il est alors facile de voir qu'un arrêt sur la courbe serait préférable, et que le cycle n'est plus optimal. Cette conclusion peut s'étendre au point $\mathbf{B}^{\star}$.

\section{E - Comparaison (1) $\rightarrow(3)$ \\ ou $(2) \rightarrow(3)$.}

On démontre les points suivants :

- Une extrémale ordinaire coupe la courbe (3) sans que le point d'intersection présente de difficulté. Ce point a été précisé par M. Gauthier [2];

- Il y a a priori quatre types de passage : $(1) \rightarrow(3)$ ou $(2) \rightarrow(3)$. Voir figure 6 . Deux sont du type :

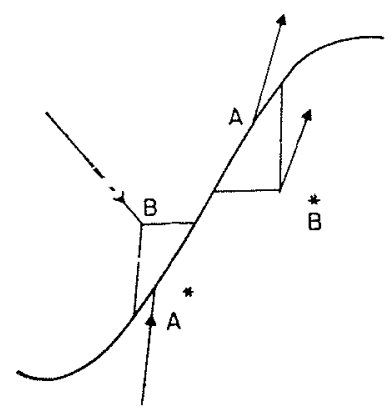

FIG. 6

$$
\begin{gathered}
\mathrm{A}, \mathrm{A}^{*} \text { avec } \mathrm{N}-\left(q-q_{0}\right) \frac{\partial \mathrm{N}}{\partial q}=0 \\
\mathbf{n} \boldsymbol{q}_{0}=-\mathrm{S} \frac{d h}{d t}
\end{gathered}
$$

Deux du type :

$$
\begin{array}{rlr}
B, B^{\star} \text { avec } N & =0 \quad \frac{\partial N}{\partial q}=0 \\
\frac{\partial n}{\partial q} & =0
\end{array}
$$

Ce sont les points $\omega_{1}$ et $\omega_{2}$ des collines $N_{1}$ et $\mathrm{N}_{2},[1]$.

La possibilité de vanner fait que l'on doil supprimer les passages $A^{*}$ et $B^{*}$. Il n'y a donc que deux types d'arrivée ou départ. 


\section{DEUXIEME PARTIE}

\section{CLASSIFICATION ET DENOMBREMENT DES CYCLES}

Nous avons jusqu'ici rencontré deux phénomènes tout à fait différents qu'il importe de bien mettre en relief :

- D'une part les trajets (1), (1'), (2), (2') et (0) s'enchaînent d'une façon « déterministe ». A des données initiales du type Cauchy correspond un trajet et un seul. Lorsqu'on franchit une frontière $\left[(\boldsymbol{1}) \rightarrow\left(\boldsymbol{1}^{\prime}\right)\right.$ par exemple], les nouvelles conditions sont déterminées de façon biunivoque. Il n'y a donc a priori aucun problème de choix de marche. D'antre part, on traversera la courbe de marée sans passer en marche (3).

Pour achever de déterminer ces trajets, nous admettrons qu'entre deux points $A$ et $B$ quelconques, ils réalisent un optimum (et non plus seulement un extremum) et que cet optimum est unique. Il en résulte bien entendu que deux courbes issues d'un point $A$ ne peuvent pas se couper.

Au contraire, les fonctionnements du type (3) interrompent cette chaîne déterministe et suppriment toute relation entre passé et avenir. Le point $A$ par lequel on quitte $\mathrm{H}=0$ est absolument indépendant du point $B$ d'arrivee sur $H=0$. Les «cycles élémentaires 》, indépendants entre eux, sont donc des cycles ( $1,1^{\prime}, 2,2^{\prime}$ et 0 ) allant de $H=0$ à $H=0$. On voit le rôle des trajets (3). Ils servent à séparer et à rendre indépendant les cycles de base. Nous pouvons d'ailleurs démontrer une proposition relative à ces trajets.

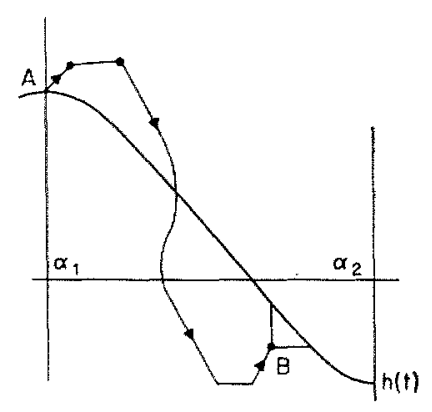

Fig. 6 bis
Proposition (6).

Soient $\left(\alpha_{0}, \alpha_{1}\right)$ et $\left(\alpha_{1}, \alpha_{2}\right)$ deux segments crêtecreux successifs, correspondant à deux branches d'un cycle de marée. Il ne peut y avoir à la fois un point A sur $\left(\alpha_{0}, \alpha_{1}\right)$ et un point B sur $\left(\alpha_{1}, \alpha_{2}\right)$ (fig. 6 bis).

Démonstration :

$$
\begin{aligned}
\text { Supposons } \frac{d h}{d t} \geqslant 0 \operatorname{sur}\left(\alpha_{0}, \alpha_{1}\right) & \\
& \frac{d h}{d t} \leqslant 0 \operatorname{sur}\left(\alpha_{1}, \alpha_{2}\right)
\end{aligned}
$$

Le départ en $A$ sera suivi d'un trajet (2) avec $\mathrm{H}>0$.

L'arrivée en $B$ sera précédée d'un trajet (2) avec $\mathrm{H}<0$.

Entre les deux, il y aura donc un point $C$ avec $\mathrm{H}=0$, et $(d \mathrm{H} / d t)<0$.

Le trajet suivi sera donc du type :

$$
A \rightarrow(2)-(0)-(1)-(0)-(2) \rightarrow B
$$

Nous aurons nécessairement deux segments d'arrêt. Or ([1] et $P_{1}$ ) le premier segment est nécessairement situé sur $\left(\alpha_{1}, \alpha_{2}\right)$. De même pour le deuxième. On contredit donc $\mathrm{P}_{5}$.

Corollaire :

S'il y a un trajet (3) sur $\left(\alpha_{0}, \alpha_{1}\right)$ et un autre sur $\left(\alpha_{1}, \alpha_{2}\right)$ ces deux trajets se raccordent: on ne quitte pas la courbe $H=0$. Ceci nous permettra de définir une méthode pour engendrer tous les cycles possibles. Mais, avant cela, nous allons préciser la structure des cycles issus d'un trajet (3).

\section{A - Structures des cycles issus d'un trajet (3).}

Soit donc un trajet (3). Nous appelons $\left(\mathrm{C}_{0}\right)$ la courbe de marée $\mathrm{H}=0$, limitée àu segment creux-crête $\left(\alpha_{0}, \alpha_{1}\right)$. Nous quitterons cette courbe par un point du type $A$, situé sur $\left(C_{0}\right)$. Nous allons étudier, pour $A_{0} \varepsilon\left(C_{0}\right)\left(^{\star}\right)$ tous les cycles qui peuvent être construits.

${ }^{\star}$ ) Note: le symbole $A \varepsilon C_{0}$ signifie: A appartient à $\left(\mathrm{C}_{3}\right)$ 


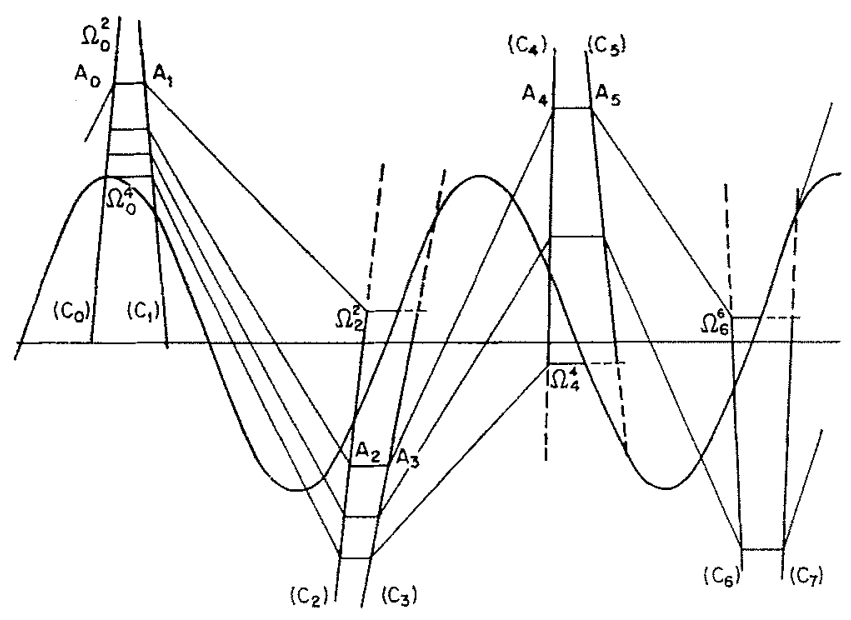

FIG. 7

Soit donc (fig. 7) un cycle ( $\mathbf{A}_{0}, \mathbf{A}_{1}, \mathbf{A}_{2}, \ldots \mathbf{A}_{n}$ ... ). Nous appelons $A_{1}$ le point de passage $(2) \rightarrow(0), A_{2}$ le point $(0) \rightarrow(1)$ etc. D'une façon générale, les points $A_{K}$ seront les points d'arrêtdépart $(1) \rightarrow(0)$ ou $(2) \rightarrow(0)$. Lorsque $A_{0}$ décrit la courbe de marée $\left(C_{0}\right)$, le point $A_{K}$ décrit une courbe $\left(\mathrm{C}_{\mathrm{K}}\right)$. Il est alors évident que :

\section{Proposition (7).}

La correspondance $\left(\mathrm{C}_{0}\right) \rightarrow\left(\mathrm{C}_{K}\right)$ est une homéomorphie biunivoque et continue.

Il suffit en effet de le démontrer pour $\left(\mathrm{C}_{\mathrm{K}}\right) \rightarrow\left(\mathrm{C}_{\mathrm{T}+1}\right)$ et de remarquer que l'on a sur $\left(\mathrm{C}_{\mathrm{K}}\right)$ comme sur $\left(\mathrm{C}_{\mathrm{K}+1}\right)$, des conditions de Cauchy.

Le fait que les trajets soient optimaux et que cet optimum soit unique va nous permettre de démontrer deux propositions :

\section{Proposition (8).}

Sur $\left(C_{\mathrm{K}}\right), Z$ (S) est monotone, les courbes $\left(\mathrm{C}_{\mathrm{K}}\right)$ ne coupent qu'une fois une horizontale donnée.

\section{Démonstration :}

Supposons (fig. 8) qu'il y ait deux points $\mathrm{M}_{\mathrm{K}}$ et $N_{K}$ situés sur la même horizontale $Z=Z_{0}$. Lorsque $A_{K}$ décrit $\left(C_{\mathrm{K}}\right)$ de $\mathrm{M}_{\mathrm{K}}$ à $\mathrm{N}_{\mathrm{K}}$, considérons l'énergie comprise entre le départ $A_{0}$ et le point d'intersection avec l'horizontale $Z=Z_{0}$. Cette énergie est maximale en $M_{K}$ et $N_{K}$. Elle passe donc par un minimum entre $\mathrm{M}_{\mathrm{KK}}$ et $\mathrm{N}_{\mathrm{KK}}$, ce qui est contradictoire.

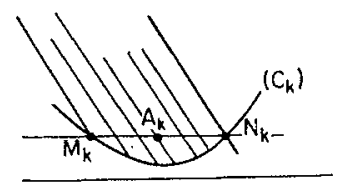

FrG. 8

\section{Proposition (9).}

Deux extrémales quelconques ne peuvent pas se couper. La démonstration est analogue à celle de $\mathrm{P}_{8}$. Remarquons d'ailleurs qu'on cesserait d'avoir un optimum unique.

Corollaire :

On peut, d'après $P_{8}$, repérer sur $\left(C_{K}\right)$ les points au moyen de leur cote $Z_{\mathrm{k}}$. Dans ces conditions $\left(Z_{\mathrm{I}}\right) \rightarrow\left(Z_{n}\right)$ est une homéomorphie. $Z_{n}$ est done une fonction monotone et même croissante de $Z_{\mathrm{K}}$ (le fait qu'elle soit croissante résulte de $P_{9}$ ). Il y a exception pour la courbe $\left(\mathrm{C}_{0}\right)$ qui est inversée par rapport aux autres).

Ainsi, nous avons montré que l'ensemble des extrémales issues de $\mathrm{C}_{0}$ balaie un domaine (D) de façon biunivoque. A un point $M$ de (D) correspond une courbe et une seule; $S_{0}$, abscisse du point de départ $A_{v}$ sur $C_{4}$ et $t$ (temps), forment donc un système de coordonnées de ce domaine. Cela est vrai également de $\left(S_{\mathrm{k}}, t\right)$ ou de $\left(Z_{K}, t\right)$. On démontre alors sans difficulté que, sur les courbes $\mathrm{C}_{\mathrm{k}}, \mathrm{H}$ est une fonction monotone (croissante) de $Z_{\mathrm{K}}$ ou de $S_{u}$.

Soit alors (fig. 7) une courbe $\left(C_{K}\right)$ correspondant par exemple au passage (1) ou (1') à $(0)$; $\left(\mathrm{C}_{\mathrm{K}}\right)$ passe en particulier par le point :

$$
\Omega_{\mathrm{K}} \mathrm{K}\left(\mathrm{N}=0 \quad \frac{\partial \mathrm{N}}{\partial q}=0 \quad \mathrm{~N}-q \frac{\partial \mathrm{N}}{\partial q}=0\right)
$$

En $\Omega_{\mathrm{K}} \mathrm{K}, \mathrm{H}=\mathrm{H}_{\mathrm{min}}$. Rappelons que si

$$
\mathrm{H}\left(\mathrm{A}_{\mathrm{K}}\right)>\mathrm{H}_{\text {win }} \text {, }
$$

le cycle comsidéré n'est plus optimal tant que

$$
\mathrm{H}\left(\mathrm{A}_{\mathrm{K}}+1\right)<0 \text {. }
$$

Donc, sur la courbe $\left(C_{\mathrm{K}}\right)$, les cycles admissibles se séparent en deux familles bien distinctes selon que :

$H\left(A_{K}\right)<H_{\text {min }}\left(A_{K}\right.$ situé au-dessous de $\left.\Omega_{K}\right)$, ou $H\left(A_{K}+1\right)>0\left(A_{K}\right.$ au-dessus de $\left.B\right)$. 


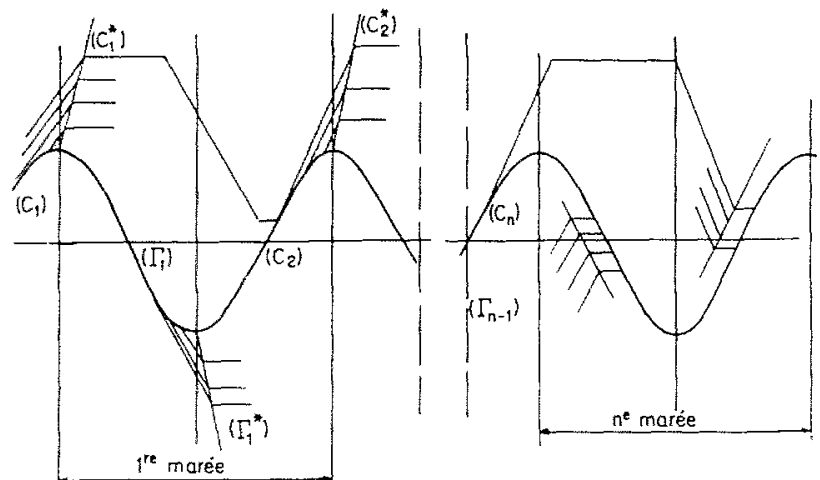

Fig. 9

Les cycles au-dessus de $B$, qui sont a priori possibles, seront considérés comme sans intérêt (il est d'ailleurs probable qu'ils seraient irréalisables pratiquement). $A_{K}$ sera donc situé en dessous de $\Omega_{\mathrm{K}} \mathrm{K}$.

Pour la même raison $A_{\mathrm{K}+2}$ sera situé au-dessus de $\Omega_{\mathrm{K}+2^{\mathrm{K}+2}}$ : donc si nous appelons :

$\Omega_{\mathrm{K}}{ }^{\mathrm{K}+2}$ l'original de $\Omega_{\mathrm{K}+2}{ }^{\mathrm{K}+2}$ sur la courbe $\left(\mathrm{C}_{\mathrm{K}}\right)$, nous voyons :

$$
\Omega_{\mathrm{K}}{ }^{\mathrm{K}+2} \leqslant \mathrm{~A}_{\mathrm{K}} \leqslant \Omega_{\mathrm{K}} \mathrm{K}
$$

De même :

$$
\Omega_{\mathrm{K}}{ }^{\mathrm{K}+2} \leqslant \mathrm{~A}_{\mathrm{K}} \leqslant \Omega_{\mathrm{K}}{ }^{\mathrm{K}+4} \leqslant \Omega_{\mathrm{K}}
$$

pour les cycles qui coupent $\left(\mathrm{C}_{\mathrm{K}+4}\right)$,

et en généralisant :

$$
\begin{aligned}
& \Omega_{\mathrm{K}} \mathrm{K}+2 \leqslant \Omega_{\mathrm{K}} \mathrm{K}+6 \ldots \leqslant \Omega_{\mathrm{K}} \mathrm{K}+2(2 n+1) \\
& \leqslant A_{K} \leqslant \Omega_{K}{ }^{K+4 n} \leqslant \ldots \leqslant \Omega_{K}^{K+4} \leqslant \Omega_{K_{K}}{ }^{K}
\end{aligned}
$$

pour les cycles qui coupent $\mathrm{C}_{\mathrm{K}+2(2 n+1)}$.

Il y aurait sans doute beaucoup d'autres résultats à démontrer concernant ces cycles et les questions de limite lorsque $n \rightarrow \infty$. Il est probable que l'on obtient ainsi une limite unique que nous appellerons $\Omega_{K \infty}{ }^{\infty}$. Cela est intéressant pour le calcul numérique des cycles, car on voit que le calcul du cycle à $n$ branches donne beaucoup de renseignements sur celui du cycle à $(n+1)$ branches.

Considérons alors (fig. 9) $n$ marées crête à crête, plus une branche ascendante $\left(C_{1}\right)$ à l'origine.

Dénombrons les cycles possibles avec départ vers le bas. Nous avons $n$ branches de départ $\left(\mathrm{C}_{1}\right)\left(\mathrm{C}_{\mathrm{s}_{2}}\right) \ldots\left(\mathrm{C}_{n}\right)$, d'où :

$$
(2 n-1)+(2 n-3)+\ldots+3+1=2 n^{2}
$$

cycles «supérieurs».
Nous avons, de même, $(n-1)$ branches de départ vers le bas soit $\left(\Gamma_{1}\right)\left(\Gamma_{2}\right) \ldots\left(\Gamma_{n-1}\right)$, d'où :

$$
(2 n-2)+(2 n-4)+\ldots+2=2 n(n-1)
$$

cycles «inférieurs».

Soit un total de :

$$
2 n[n+(n-1)]==^{\prime} 2 n(2 n-1) \text { cycles. }
$$

Tous les cycles sont des combinaisons de ceuxlà. Le problème de.calcul proprement dit -.. $2 n(2 n+1)$ cycles élémentaires - cède la place à un problème de combinaison.

Nous allons donc dénombrer les combinaisons possibles. Soient par exemple $n$ marées successives. Numérotons de 1 à $2 n$ les segments crêtecreux. A chaque segment, associons :

le nombre 0 s'il n'y a pas de trajet de type (3), le nombre 1 s'il y a un trajet de type (3).

Convenons, de plus, pour éviter les problèmes de bord, que la branche ascendante 0 et la branche descendante $2 n+1$ auront le chiffre 1 , c'esta-dire correspondront à des trajets (3). Il est alors facile de voir que à toute suite binaire à $2 n$ termes, correspond un cycle de marée unique, et réciproquement.

Nous pouvons donc fabriquer $2^{2 n}=4^{n}$ cycles possibles.

Dénombrons maintenant les cycles supérieurs. Nous avons $n$ marées. A une marée quelconque, de rang $K$ par exemple, correspondent les quatre possibilités :

$$
\begin{array}{ll}
00 & 10 \\
01 & 11
\end{array}
$$

De ces quatre possibilités, la troisième est à éliminer, car elle correspond à un démarrage vers le bas. Nous avons donc $3^{n}$ cycles supérieurs possibles.

Nous pouvons enfin remarquer que les extrémales correspondant à des trajets (3) débordant sur deux branches (ou plus) qui sont donc représentées par des suites du type 0110, 01110, 011110, etc, sont d'un intérêt médiocre. Nous pouvons donc nous intéresser aux autres. Appelons $\mathrm{N}_{K}$ le nombre de ces suites ( $\mathrm{K}$ désignant le numéro de la branche).

On trouve facilement que l'on a la relation récurrente :

$$
N_{(\mathrm{K})}=\mathbf{N}_{(\mathrm{K}-1)}+\mathbf{N}_{(\mathrm{K}-2)}
$$


d'où la suite :

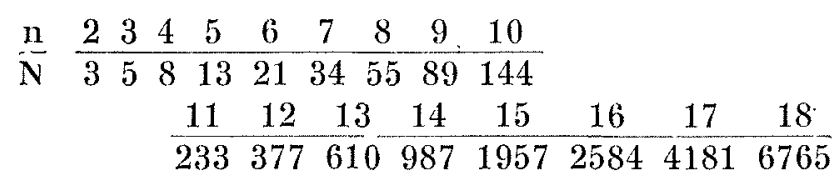

Restreignons encore le problème en cherchant les suites «supérieures» qui ne représentent pas de répétitions.

Il est alors évident que, pour une marée nous n'avons plus que les deux possibilités $(0,0)$ et $(0,1)$, et que ceci élimine bien des répétitions. D'où $2^{n}$ cycles possibles.

Pour conclure, nous donnons le tableau des chiffres obtenus :

\begin{tabular}{|c|c|}
\hline 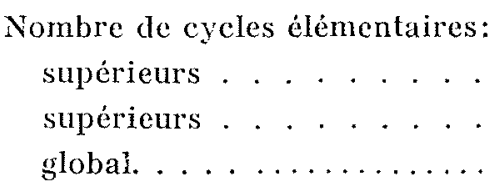 & $\begin{array}{l}2 n^{2} \\
2 n(n+1) \\
2 n(2 n+1)\end{array}$ \\
\hline $\begin{array}{l}\text { Nombre de cycles composés : } \\
\text { global. } \ldots \ldots \ldots \ldots \ldots \ldots \\
\text { supérieurs } \ldots \ldots \ldots \ldots \ldots \ldots \\
\text { sans répétition. } \ldots \ldots \ldots \ldots \\
\text { supérieurs sans répétition.. }\end{array}$ & $\mathrm{N} \sim \mathrm{A}\left(\frac{1+\sqrt{5}}{2}\right)^{3^{n}}$ \\
\hline
\end{tabular}

\section{BIBLIOGRAPHIE}

[1] R. Gibrat. - L'énergie des marées, Bulletin S.F.E., $7^{\text {V }}$ série, III, mai 1953 , pp. 283-332.
[2] M. Gauther. - Communication présentée à la S.H.F., séance du 16 mars 1962.

Les notations utilisées sont les mèmes que celles de ces deux articles.

\section{I S CUSSION}

Président: M. Yvon

M. le Président souligne l'importance de l'effort qui a été accompli pour essayer d'appréhender les points qui restent mystérieux dans l'étude des cycles des usines marémotrices.

Le travail de M. GaUthien est surtout très important pour connaître les points de transition et il est extrếmement remarquable que l'ordre de grandeur trouvé par le calcul pour faire les transitions, sans connaitre la machine, corresponde à peu près à l'ordre de grandeur acceptable pour la machine.

Le travail de M. CaSeau est aussi, de première importance. Les conséquences n'en sont pas encore complètement tirées quant à la possibilité de comprendre les cycles à très long terme et pour savoir comment est influencée cette fameuse marche 3 qui, passant par un point exceptionnel, réintroduit le choix que le mécanisme avait supprimé.

M. Duroux demande si l'on a cherché à se rendre compte de la perte de productivité qui intervient lorsque les cycles réalisés s'écartent dans une certaine mesure des cycles optimaux. En prineipe, comme ces derniers résultent d'un calcul des variations, la perte de productivité devrait être un infiniment petit d'ordre supérieux par rapport aux écarts du cycle, supposés petits ou modérés.

La question ponlrait présenter un certain intérêt pratique parce que les «collines » caractéristiques des machines, sur lesquelles s'appuie le calcul des variations, ne sont pas exactement connues, et surtout évolueront dans le temps avec l'usure des machines.

II. CasEau répond que :

- d'une part, les écarts possibles sont d'autant plus faibles que les points sont rapprochés;

- d'autre part, on essaiera d'utiliser au mieux le cycle

limite, mais on ne pourra estimer l'importance des

ćarts que lorsque l'on aura des idées plus précises

sur le cycle limite qui sera adopté pour la Rance.

M. Ie Président pense que les écarts seront de quelques ponr cent, si entre deux points $A$ et $B$ de la sinusoïde, on part bien au bon moment et on arrive bien au bon moment, même en suivant une intégrale différente entre ces deux moments; par contre, si l'on dit à un enfant de construire la ligne qui lui plaît pour joindre les deux points, l'erreur est systematique et on pent perdre $80 \%$ de l'énergie.

Il $y$ a des écarts que l'on peut très bien connaître : sur un simple effet, parfaitement défini, si l'on part un quart d'heure trop tôt ou trop tard, on perdra $3 / 4$ de l'énergie.

En somme, un certain nombre de pelites erreurs autour d'une bonne courbe m'a pas d'importance; mais lorsque l'erreur est systématique, on arrive rapidement à lout perdre.

Un bon dessinateur qui aura pris l'habitude de ces calculs d'énergie et qui a conscience de lenr importance travaillera $\grave{a} 2 \%$ près. L'introduction d'une machine sur une marée ne pourra donc faire gagner plus de quelques pour cent.

En ce qui concerne l'évolution des caractéristiques dans le temps avec l'usure des machines, M. Ie Président indique que des vérifications seront failes périodiquement au cours de l'exploitation en vae de rajustor les cycles aux nouvelles caractéristiques.

M. le Président ajoule que le but des átudes présentées par MM. Caseau et Gauther n'est pas seulement d'obtenir un gain de $2 \%$ sur la productivile mais ac chercher a acomprendre» Je problème de cette énergic nouvelle et d'éviter tonte surprise dans son exploitat tion: «Nous arons dejà compris beaucoup de choses depuis deux ans et les annees de fin de construction de l'usine de la Rance nous laissent encore une certaine chance d'accroitre notre connaissance.»

M. Caseau indique que la forme du bassin de la Rance ne se modifiera pas, el qu'il y atra done unc partie de l'équation qui restera bien connuc, mais qu'on ne pent pas encore savoir si les écarts possibles entre la courbe $z$ (t) résullant des études actuelles et l'énergie qui se manifestera permettront d'amcliorer les calculs précédents. Il croit done qu'il faudra se contenter de la colline moyenne, tant que l'on n'aura pas précise l'origine des erreurs qui peurent provenir d'une comnas. sance imparfaite de la marée, des remous, elc... Entre temps, il ne lui parait pas ulile de refaire des «collines $\gg$ en se reportant à la machine.

II. Ie Président remereic les menbres présents de leur attention sur ces sujets difficiles et se félicite de voir qu'ils intéressent beaucoup d'entre eux. 